\title{
Multi-Branch Tomlinson-Harashima Precoding for MU-MIMO Systems: Theory and Algorithms
}

\author{
Keke Zu, Rodrigo C. de Lamare, Senior Member, IEEE and Martin Haardt, Senior Member, IEEE
}

\begin{abstract}
Tomlinson-Harashima precoding (THP) is a nonlinear processing technique employed at the transmit side and is a dual to the successive interference cancelation (SIC) detection at the receive side. Like SIC detection, the performance of THP strongly depends on the ordering of the precoded symbols. The optimal ordering algorithm, however, is impractical for multiuser MIMO (MU-MIMO) systems with multiple receive antennas due to the fact that the users are geographically distributed. In this paper, we propose a multi-branch THP (MB-THP) scheme and algorithms that employ multiple transmit processing and ordering strategies along with a selection scheme to mitigate interference in MU-MIMO systems. Two types of multi-branch THP (MB-THP) structures are proposed. The first one employs a decentralized strategy with diagonal weighted filters at the receivers of the users and the second uses a diagonal weighted filter at the transmitter. The MB-MMSE-THP algorithms are also derived based on an extended system model with the aid of an LQ decomposition, which is much simpler compared to the conventional MMSE-THP algorithms. Simulation results show that a better bit error rate (BER) performance can be achieved by the proposed MB-MMSE-THP precoder with a small computational complexity increase.
\end{abstract}

Index Terms-Multiuser MIMO (MU-MIMO), TomlinsonHarashima precoding (THP), multi-branch (MB).

\section{INTRODUCTION}

\section{A. Background and Problem Formulation}

Multi-user MIMO (MU-MIMO) systems are promising for downlink wireless transmissions since they can improve the average user spectral efficiency [1]. When channel state information (CSI) is available at the transmit side, precoding techniques can be employed at the base station (BS) to mitigate the Multiuser Interference (MUI). Then, the required computational effort for each user's receiver can be reduced and eventually the receiver structure can be simplified [2]. For these reasons, the design of cost-effective precoders is particularly important for the downlink of MU-MIMO systems.

Channel inversion based linear precoding techniques such as zero forcing (ZF) and minimum mean squared error (MMSE) precoding [3] - [5] are attractive due to their simplicity. However, channel inversion based precoding techniques require a higher average transmit power than other precoding algorithms especially for ill conditioned channel matrices, which could result in a reduced bit error ratio (BER) performance [4]. As a generalization of $\mathrm{ZF}$ precoding, block diagonalization (BD) based precoding algorithms have been proposed in [6], [7] for MU-MIMO systems. However, BD based precoding algorithms only take the MUI into account and thus suffer a performance loss at low signal to noise ratios (SNRs)

Parts of this work have been published at the ITG/IEEE Workshop on Smart Antennas, Dresden, Germany, Mar. 2012 (see reference [29]). when the noise is the dominant factor. A regularized block diagonalization (RBD) precoding algorithm which introduces a regularization factor to take the noise term into account has been proposed in [8]. The performance is improved by RBD precoding, but the BD-type precoding algorithms still cannot achieve the maximum transmit diversity. A nonlinear vector perturbation (VP) approach, which is based on sphere encoding (SE) to perturb the data, was proposed in [9]. With the perturbation, a near optimal performance is achieved by VP precoding. However, finding the optimal perturbation vector can be a nondeterministic polynomial time (NP)-hard problem.

\section{B. Prior Art}

Another nonlinear and data-modifying technique is the dirty paper coding (DPC) proposed in [10]. It was shown that the capacity of systems using DPC with independent and identically distributed (i.i.d.) Gaussian interference is equal to that of interference-free systems. However, DPC is not suitable for practical use due to the requirement of infinitely long codewords [11]. Tomlinson-Harashima precoding (THP) [12], [13] is a pre-equalization technique originally proposed for channels with intersymbol interference (ISI). Then, the THP technique was extended from temporal equalization to spatial equalization for MIMO precoding in [14]. The details of THP algorithms are illustrated in Section II. Although THP suffers a performance loss compared to DPC as shown in [15], it can work as a cost-effective replacement of DPC in practice [16]. As reported in [14], [17], the THP structure can be seen as the dual of successive interference cancelation (SIC) detection implemented at the receive side. Like SIC detection, the performance of THP systems strongly depends on the ordering of the precoded symbols.

A V-BLAST like ordering strategy for THP has been studied in [18] - [20]. The V-BLAST ordering requires multiple calculations of the pseudo inverse of the channel matrix. Therefore, a suboptimal heuristic sorted LQ decomposition algorithm has been extended from the sorted QR decomposition in [21], [22] to THP and a tree search (TS) algorithm has also been proposed in [24]. Researchers in [23], [25] noticed the importance of the ordering to the THP performance as well, and the best-first ordering approach has been proposed to perform the ordering. Algorithms for finding the nearoptimal order are proposed in [26], [27]. The above ordering algorithms, however, assume that each distributed receiver is equipped with a single antenna. Therefore, cooperative ordering processing is impractical for distributed receivers with multiple antennas. In [28], a successive optimization THP (SO-THP) algorithm has been proposed for users with multiple 
antennas, but SO-THP only offers a small BER gain over THP at low SNRs. For high SNRs, the BER performance of SOTHP is comparable to that achieved by the conventional THP algorithm. In order to achieve a better BER performance in the whole SNR range, a novel THP structure is proposed in this work based on a multiple-branch (MB) strategy for MU-MIMO systems with multiple antennas at each receiver. Although the MB-THP structure for single-user MIMO (SUMIMO) systems has been studied in [29], the original structure cannot be applied to MU-MIMO systems since the users are physically distributed.

\section{Contributions}

In the literature, there are two basic THP structures according to the position of the diagonal weighted filters, decentralized filters located at the receivers or centralized filters deployed at the transmitter, which are denoted as dTHP or cTHP, respectively [30]. Most of the previous research works on THP, however, have only focused on one of the structures. In this work, we develop MB-THP techniques for both of the two basic THP structures. We derive the MMSE precoding filters using an LQ decomposition. Then, we present a design strategy for the transmit patterns that implements an effective ordering of the data streams along with a selection criterion for the best pattern. An analysis and a comparison between MB-dTHP and MB-cTHP are also illustrated. By utilizing the MB strategy, the transmit diversity gain is maximized for MU-MIMO systems with spatial multiplexing. Therefore, the final BER performance is improved by the proposed MBTHP algorithms. The main contributions of the work can be summarized as

1) Novel MB-THP algorithms are developed based on two basic THP structures.

2) Cost-effective MMSE filters are derived based on the LQ decomposition of an extended matrix along with the design of transmit patterns and a selection procedure.

3) A comprehensive performance analysis is carried out in terms of the error covariance matrix, the sum-rate and the computational complexity.

4) A study of the most relevant precoding algorithms reported in the literature and the proposed MB-THP algorithm is conducted.

This paper is organized as follows. The system model and the basics of THP techniques are described in Section II. The proposed MB-THP scheme and algorithms are described in detail in Section III. A performance analysis of the existing and proposed precoders is developed in Section IV. Simulation results and conclusions are presented in Section V and Section VI, respectively.

\section{System Model ANd THP Algorithms}

We consider an uncoded MU-MIMO broadcast channel, with $N_{t}$ transmit antennas at the base station (BS) and $N_{k}$ receive antennas at the $k$ th user equipment (UE). With $K$ users in the system, the total number of receive antennas is $N_{r}=\sum_{k=1}^{K} N_{k}$. When $N_{r}=N_{t}$, the channel matrix is a square matrix. When $N_{r} \geq N_{t}$, a scheduling procedure is first performed to generate a square equivalent channel matrix. The total number of transmitted streams is denoted by $S$, and the channel is assumed to be always a square matrix, that is $\boldsymbol{H}=\left[\boldsymbol{H}_{1}^{T}, \boldsymbol{H}_{2}^{T}, \cdots, \boldsymbol{H}_{K}^{T}\right]^{T} \in \mathbb{C}^{S \times S}$ is the combined channel matrix and $\boldsymbol{H}_{k} \in \mathbb{C}^{N_{k} \times S}$ is the $k$ th user's channel matrix. Note that power-loading schemes [17] could be used to determine the number of data streams or allocate more power to a weaker user to improve the overall performance. However, for simplicity, we assume that all data streams are active and equal power loading between users and streams is performed since the power allocation is not the focus of this paper.

\section{A. Two Basic THP Structures}

Based on the knowledge of CSI at the transmit side, the interference of the parallel streams of a MIMO system with spatial multiplexing can be subtracted from the current stream. This SIC technique at the transmit side is known as THP and can be seen as the dual of SIC detection at the receive side. Generally, there are three filters to implement THP algorithms: the feedback filter $\boldsymbol{B} \in \mathbb{C}^{S \times S}$, the feedforward filter $\boldsymbol{F} \in \mathbb{C}^{S \times S}$, and the scaling matrix $\boldsymbol{G} \in \mathbb{C}^{S \times S}$. According to the position of $\boldsymbol{G}$, there are two basic THP structures, which are illustrated in Fig. 1. The decentralized THP (dTHP) employs $\boldsymbol{G}$ (or sub-matrices of it) at the receivers, whereas the centralized THP (cTHP) uses $\boldsymbol{G}$ at the transmitter.

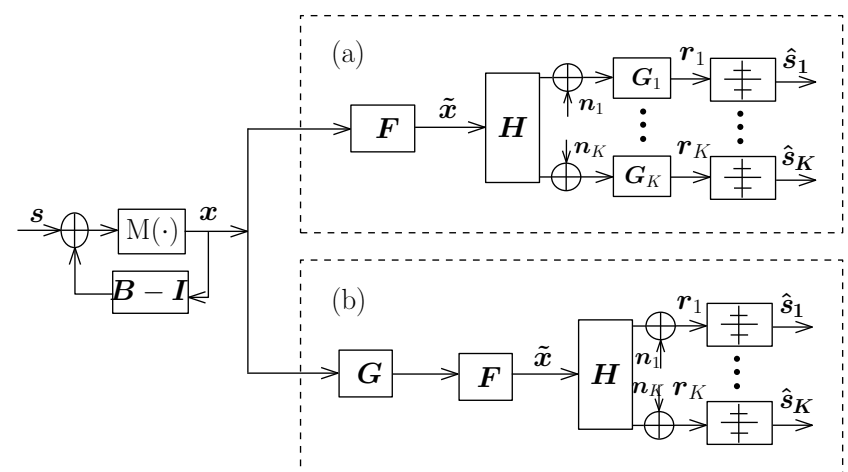

Fig. 1: The two basic THP structures

(a) Decentralized THP: the scaling matrix $G$ is separately placed at the receivers.

(b) Centralized THP: the scaling matrix $G$ is placed at the transmitter.

The feedback filter $\boldsymbol{B}$ is used to successively cancel the interference caused by the previous streams from the current stream. Therefore, the feedback filter $\boldsymbol{B}$ should be a lower triangular matrix with ones on the main diagonal [17]. The feedforward filter $\boldsymbol{F}$ is used to enforce the spatial causality and has to be implemented at the transmit side for MU-MIMO systems because the physically distributed users cannot be processed jointly. The scaling filter $G$ contains the corresponding weighted coefficient for each stream and thus it should have a diagonal structure. The quantity $\boldsymbol{x} \in \mathbb{C}^{S \times 1}$ is the combined transmit signal vector after the feedback operation and $\tilde{\boldsymbol{x}}$ is the combined transmit signal vector after precoding, $\tilde{\boldsymbol{x}}=\boldsymbol{F} \boldsymbol{x}$ for dTHP and $\tilde{\boldsymbol{x}}=\boldsymbol{F} \boldsymbol{G} \boldsymbol{x}$ for cTHP. Finally, the received signal after the feedback, feedforward, and the scaling filter, for the dTHP and cTHP is respectively given by

$$
\boldsymbol{r}^{(\mathrm{dTHP})}=\boldsymbol{G}(\boldsymbol{H} \boldsymbol{F} \boldsymbol{x}+\boldsymbol{n}),
$$




$$
\boldsymbol{r}^{(\mathrm{cTHP})}=\beta\left(\boldsymbol{H} \cdot \frac{1}{\beta} \boldsymbol{F} \boldsymbol{G} \boldsymbol{x}+\boldsymbol{n}\right),
$$

where the quantity $\boldsymbol{n}=\left[\boldsymbol{n}_{1}^{T}, \boldsymbol{n}_{2}^{T}, \cdots, \boldsymbol{n}_{K}^{T}\right]^{T} \in \mathbb{C}^{S \times 1}$ is the combined Gaussian noise vector with i.i.d. entries of zero mean and variance $\sigma_{n}^{2}$. The factor $\beta$ is used to impose the power constraint $\mathrm{E}\|\tilde{\boldsymbol{x}}\|^{2}=\xi$ with $\xi$ being the average transmit power.

\section{B. Review of THP Algorithms}

As reported in the literature, SIC detection can be efficiently implemented by a QR decomposition [31], whereas THP can be implemented by an LQ decomposition. By utilizing an LQ decomposition on the channel matrix $\boldsymbol{H}$, we have

$$
\boldsymbol{H}=\boldsymbol{L} Q,
$$

where $L$ is a lower triangular matrix and $Q$ is a unitary matrix (by unitary we mean $\boldsymbol{Q}^{H} \boldsymbol{Q}=\boldsymbol{Q} Q^{H}=\boldsymbol{I}$ ). Therefore, the filters for the THP algorithm can be obtained as

$$
\begin{array}{r}
\boldsymbol{F}=\boldsymbol{Q}^{H}, \\
\boldsymbol{G}=\operatorname{diag}\left[l_{1,1}, l_{2,2}, \cdots, l_{S, S}\right]^{-1}, \\
\boldsymbol{B}^{(\mathrm{dTHP})}=\boldsymbol{G} \boldsymbol{L}, \boldsymbol{B}^{(\mathrm{cTHP})}=\boldsymbol{L} \boldsymbol{G},
\end{array}
$$

where $l_{i, i}$ is the $i$ th diagonal element of the matrix $\boldsymbol{L}$.

From Fig. 1 the transmitted symbols $x_{i}$ are successively generated as

$$
x_{i}=s_{i}-\sum_{j=1}^{i-1} b_{i, j} x_{j}, i=1, \cdots, S,
$$

where $s_{i}$ is the $i$ th transmit data with variance $\sigma_{s}^{2}$ and $b_{i, j}$ are the elements of $\boldsymbol{B}$ in row $i$ and column $j$. From the above formulation, the transmit power will be significantly increased as the amplitude of $x_{i}$ exceeds the modulation boundary by the successive cancelation. In order to reduce the amplitude of the channel symbol $x_{i}$ to the boundary of the modulation alphabet, a modulo operation $\mathrm{M}(\cdot)$ should be employed which is defined element-wise as [33]

$$
\mathrm{M}\left(x_{i}\right)=x_{i}-\left\lfloor\frac{\operatorname{Re}\left(x_{i}\right)}{\tau}+\frac{1}{2}\right\rfloor \tau-j\left\lfloor\frac{\operatorname{Im}\left(x_{i}\right)}{\tau}+\frac{1}{2}\right\rfloor \tau,
$$

where $\tau$ is a constant for the periodic extension of the constellation. The specific value of $\tau$ depends on the chosen modulation alphabet. Common choices for $\tau$ are $\tau=2 \sqrt{2}$ for QPSK symbols and $\tau=8 \sqrt{10}$ in case of rectangular 16-QAM when the symbol variance is one [33]. The modulo processing is equivalent to adding a perturbation vector $\boldsymbol{d}$ to the transmit data $s$, such that the modified transmit data are [20]

$$
v=s+d .
$$

Thus, the initial signal constellation is extended periodically and the effective $k$ th transmit data symbols $\boldsymbol{v}_{k}$ are taken from the expanded set.

Although the modulo operation is employed to restrict the amplitude of $\boldsymbol{x}$ within the same scale as that of $s$, a power loss is introduced by the nonlinear processing of THP, which can be measured by $\alpha=\frac{M}{M-1}$ for the M-QAM constellations [15], [17]. The power loss is not negligible for small modulation sizes, but for moderate sizes of $M$ it is negligible and vanishes as $M$ increases. Except for the power loss, a modulo loss is also introduced by THP due to the received symbols at the boundary of a constellation may be mistaken for symbols at the opposite boundary [15]. The modulo loss is more significant for the small constellations. We neglect the power and modulo loss in this work since moderate sizes of $M$ are employed. Then, we have $\mathrm{E}\|\boldsymbol{x}\| \approx \mathrm{E}\|\boldsymbol{s}\|$. Since the statistical property of $\boldsymbol{x}$ is not changed by the multiplication of the unitary matrix $\boldsymbol{F}$, the normalization factor $\beta$ is not necessary for dTHP. For cTHP, since the power and modulo loss can be neglected, the normalization factor is approximately obtained as

$$
\beta=\frac{\mathrm{E}\|\boldsymbol{F} \boldsymbol{G} \boldsymbol{x}\|}{\mathrm{E}\|\boldsymbol{s}\|} \approx \sqrt{\sum_{i=1}^{S}\left(1 / l_{i, i}^{2}\right)} .
$$

Mathematically, the feedback processing is equivalent to an inversion operation $\boldsymbol{B}^{-1}$. Therefore, the transmitted symbol $\boldsymbol{x}$ can be written as

$$
\boldsymbol{x}=\boldsymbol{B}^{-1} \boldsymbol{v}=\boldsymbol{B}^{-1}(\boldsymbol{s}+\boldsymbol{d}),
$$

Then, the received signal for dTHP and cTHP can be respectively expressed as

$$
\begin{aligned}
& \boldsymbol{r}^{(\mathrm{dTHP})}=\boldsymbol{v}+\boldsymbol{G} \boldsymbol{n}, \\
& \boldsymbol{r}^{(\mathrm{\textrm {THHP }})}=\boldsymbol{v}+\beta \boldsymbol{n} .
\end{aligned}
$$

\section{Proposed MB-THP Precoding Algorithm}

In this section, we first analyze the interference of the two basic THP structures and show that the ordering of the precoded symbols plays an important role for both of them. Based on this analysis, the structure of the MB-cTHP and MB-dTHP precoding techniques are proposed and illustrated. Especially for the MU-MIMO setting with multiple receive antennas a cost-effective transmit pattern is developed, and a selection criterion is also deduced for both of the MBcTHP and MB-dTHP algorithms. Finally, since the MMSETHP structures are the main focus of this paper, filters for MB-MMSE-cTHP and MB-MMSE-dTHP are derived based on an extended system model which is much simpler from a computational point of view, as compared to conventional MMSE-THP techniques reported in the literature so far.

\section{A. Motivation of the Proposed MB-THP Algorithm}

As shown in equations (12) and (13), the MU-MIMO channel is decomposed into parallel additive white Gaussian noise (AWGN) channels by the successive THP processing. With the power and modulo loss ignored, the power of $\boldsymbol{v}$ is approximately equal to that of $s$. Then, the error covariance matrices of the effective transmit signal $v$ for dTHP and cTHP schemes are respectively given by

$$
\begin{aligned}
& \boldsymbol{\Phi}_{\mathrm{dTHP}}=\operatorname{diag}\left(\sigma_{n}^{2} / l_{1,1}^{2}, \cdots, \sigma_{n}^{2} / l_{S, S}^{2}\right) \\
& \boldsymbol{\Phi}_{\mathrm{cTHP}}=\operatorname{diag}\left(\sigma_{n}^{2} \sum_{i=1}^{S}\left(1 / l_{i, i}^{2}\right), \cdots, \sigma_{n}^{2} \sum_{i=1}^{S}\left(1 / l_{i, i}^{2}\right)\right)(
\end{aligned}
$$

From (14) and (15), we can verify that the error covariance matrices are different among layers for dTHP while they 
are equal for cTHP. Therefore, for each layer, the SNR is inversely proportional to $1 / l_{i, i}^{2}$ for dTHP, while it is inversely proportional to $\sum_{i=1}^{S}\left(1 / l_{i, i}^{2}\right)$ for cTHP. Due to the lower triangular structure of the feedback matrix $\boldsymbol{B}$, the interference from the transmitted data $s_{1}, s_{2}, \cdots, s_{S}$ is canceled out from $s_{1}$ to $s_{S}$ in dTHP. That is, the layer precoded first will interfere with the layer precoded afterward. Then, the performance of dTHP will be dominated by the layer with the minimum SNR. For cTHP, the sum $\sum_{i=1}^{S}\left(1 / l_{i, i}^{2}\right)$ can be influenced by reordering the rows of $\boldsymbol{H}$ during the LQ decomposition. It is worth noting that the row pivoting known in mathematics is used for the LQ decomposition when a matrix $\boldsymbol{B}$ is rank deficient, that is

$$
P B=L Q
$$

where the row permutation matrix $\boldsymbol{P}$ is chosen so that the diagonal elements of $|\boldsymbol{L}|$ are decreasing with $|\cdot|$ being the element-wise absolute value operation. The specific requirement of the row permutation matrix $\boldsymbol{P}$ does not take the physical location of the receive antennas into account, which prohibits the application of the row pivoting scheme in MUMIMO systems with multiple receive antennas since the data streams that belong to one user may be allocated to other users. For the special case when all distributed users are equipped with a single antenna, the row permutation matrix $\boldsymbol{P}$ needs to be calculated for each transmission when the channel changes to ensure a decreasing order.

In particular, the ordering of the precoded symbols plays an important role in the performance of THP systems. Thus, considerable research efforts have been spent on the development of various ordering methods [18] - [27]. However, they all focused on SU-MIMO or MU-MIMO systems with single receive antenna. For MU-MIMO systems with multiple receive antennas, these cooperative ordering algorithms are impractical due to the geographically distributed users. In addition, most of the ordering algorithms only consider one THP structure, either cTHP or dTHP.

In this work, a MB-THP structure with efficient transmit pattern design, which is predefined and especially suited for the users equipped with multiple antennas, is proposed based on the two basic THP structures. The design of transmit patterns is performed in two steps. In the first step we get the ordering patterns $\boldsymbol{T}_{u}^{(i)}$ for $i=1, \cdots, K$ between multiple users. In the second step, we obtain the ordering patterns $\boldsymbol{T}_{k_{i}}^{(j)}$ between multiple streams for the $i$ th user with $j$ denoting the different ordering states.

\section{B. Structure of the Proposed MB-THP}

The idea of multi-branch (MB) processing has been first proposed in [34] as the parallel arbitrated branches to improve the performance of decision feedback (DF) receivers. MBSIC detectors have been proposed in [35], [37] to exploit diversity gains in MIMO systems. In [36], the authors applied the MB strategy to generate interleaving patterns for DSCDMA systems. Inspired by these research works, the MBTHP algorithms for the MU-MIMO downlink are developed and proposed in this work. The structures of the proposed
MB-THP schemes are illustrated in Fig. 2. The matrices $\boldsymbol{T}^{(l)} \in \mathbb{C}^{N_{r} \times N_{r}}\left(l=1, \cdots, L_{B}\right)$ are the transmit patterns used to generate multiple parallel candidate branches, where $L_{B}$ is the total number of branches. A proper selection metric is employed to choose the optimal branch to transmit the data streams. Then, the matrices $\boldsymbol{B}^{(o)}, \boldsymbol{F}^{(o)}$ and $\boldsymbol{G}^{(o)}$ represent the feedback, feedforward and scaling filters for the selected branch.

\section{Design of the Transmit Patterns}

One of the objectives of this work is to design transmit patterns that are effective and simple. Observing the formulation in (14) and (15), the SNR performance of dTHP and cTHP can be influenced by $\boldsymbol{\Phi}_{\mathrm{dTHP}}$ and $\boldsymbol{\Phi}_{\mathrm{cTHP}}$. An ordering of the rows of $\boldsymbol{H}$ will lead to a corresponding change of $\boldsymbol{L}$ and $\boldsymbol{\Phi}$. Therefore, different ordering patterns can be employed to generate multiple branches for exploiting extra transmit diversity gains. Motivated by this, we pre-store the designed transmit patterns both at the transmitter and the receivers, which means that they are known permutations. Drawing upon previous design methods in [34] and [35], and considering the nature of distributed users in MU-MIMO scenarios, the design of transmit patterns is developed in three steps.

As the total number of users is $K$, we first obtain the different ordering patterns $\boldsymbol{T}_{u}^{(i)}$ between multiple users by

$$
\begin{array}{r}
\boldsymbol{T}_{u}^{(1)}=\boldsymbol{I}_{K}, \\
\boldsymbol{T}_{u}^{(i)}=\left[\begin{array}{cc}
\boldsymbol{I}_{p} & \mathbf{0}_{p, K-p} \\
\mathbf{0}_{K-p, p} & \boldsymbol{\Pi}_{K-p}
\end{array}\right], 2 \leq i \leq K,
\end{array}
$$

where $p=(i-2)$ and $\boldsymbol{\Pi}_{K-p}$ denotes the exchange matrix of size $(K-p) \times(K-p)$ with ones on the reverse diagonal and the superscript $i$ in $\boldsymbol{T}_{u}^{(i)}$ is termed as the ordering state. For the $K=3$ case, we have

$$
\boldsymbol{T}_{u}^{(1)}=\left[\begin{array}{lll}
1 & 0 & 0 \\
0 & 1 & 0 \\
0 & 0 & 1
\end{array}\right], \boldsymbol{T}_{u}^{(2)}=\left[\begin{array}{lll}
0 & 0 & 1 \\
0 & 1 & 0 \\
1 & 0 & 0
\end{array}\right], \boldsymbol{T}_{u}^{(3)}=\left[\begin{array}{lll}
1 & 0 & 0 \\
0 & 0 & 1 \\
0 & 1 & 0
\end{array}\right] .
$$

Next, in order to make the branches as non-contiguous as possible, we shuffle the streams for each user in a similar way. The ordering patterns for the $k$ th user equipped with $N_{k}$ receive antennas is given by

$$
\begin{array}{r}
\boldsymbol{T}_{s_{k}}^{(1)}=\boldsymbol{I}_{N_{k}}, \\
\boldsymbol{T}_{s_{k}}^{(j)}=\left[\begin{array}{cc}
\boldsymbol{I}_{q} & \mathbf{0}_{q, N_{k}-q} \\
\mathbf{0}_{N_{k}-q, q} & \boldsymbol{\Pi}_{N_{k}-q}
\end{array}\right], 2 \leq j \leq J,
\end{array}
$$

where $q=(j-2)$ and $J$ is the maximum number of ordering states. Assuming that the first, second, and third user are equipped with 2,2 , and 3 receive antennas, respectively, then, we have

$$
\begin{gathered}
\boldsymbol{T}_{s_{1}}^{(1)}=\boldsymbol{T}_{s_{2}}^{(1)}=\left[\begin{array}{ll}
1 & 0 \\
0 & 1
\end{array}\right], \boldsymbol{T}_{s_{1}}^{(2)}=\boldsymbol{T}_{s_{2}}^{(2)}=\left[\begin{array}{ll}
0 & 1 \\
1 & 0
\end{array}\right], \\
\boldsymbol{T}_{s_{3}}^{(1)}=\left[\begin{array}{lll}
1 & 0 & 0 \\
0 & 1 & 0 \\
0 & 0 & 1
\end{array}\right], \boldsymbol{T}_{s_{3}}^{(2)}=\left[\begin{array}{lll}
0 & 0 & 1 \\
0 & 1 & 0 \\
1 & 0 & 0
\end{array}\right], \boldsymbol{T}_{s_{3}}^{(3)}=\left[\begin{array}{lll}
1 & 0 & 0 \\
0 & 0 & 1 \\
0 & 1 & 0
\end{array}\right] .
\end{gathered}
$$

Unlike the ordering states in $\boldsymbol{T}_{u}^{(i)}$, the total number of ordering states in $\boldsymbol{T}_{s_{k}}^{(j)}$ for each user is not uniform. We first select the 


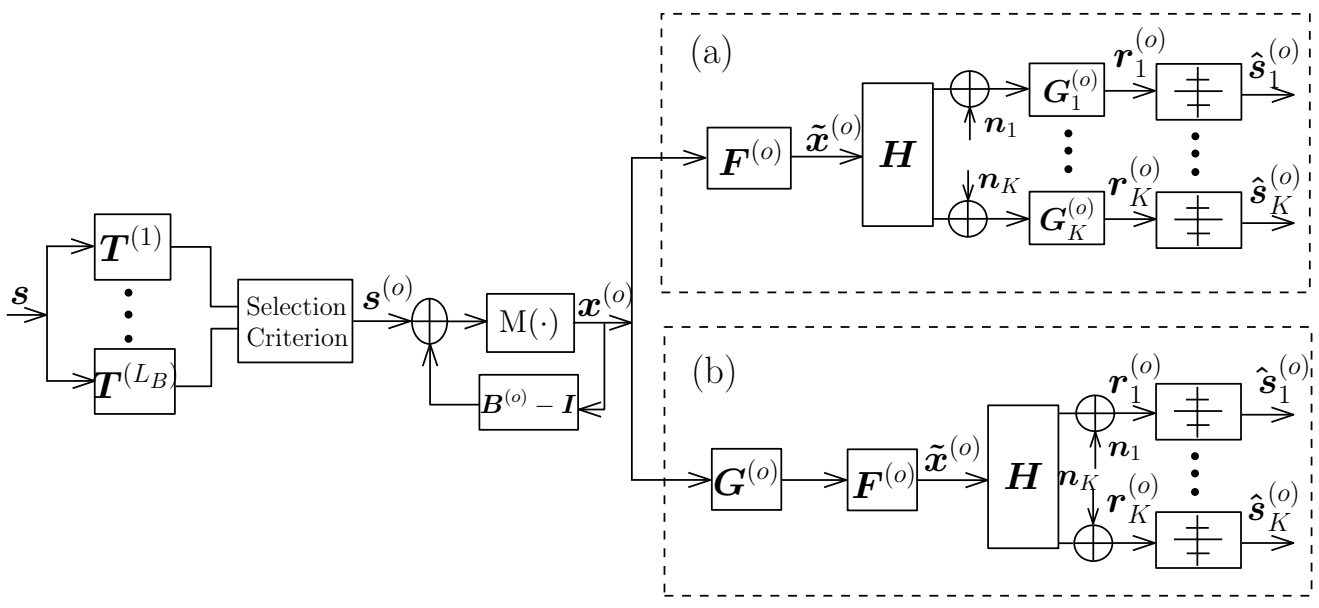

Fig. 2: The proposed MB-THP structures (a) MB-dTHP (b) MB-cTHP

user with the maximum number of receive antenna, which is equal to the maximum ordering states, i.e., $J=\operatorname{Max}_{k}\left(N_{k}\right)$ but we note that different strategies for choosing $J$ are possible.

Finally, we need to package the two ordering patterns $\boldsymbol{T}_{u}^{(i)}$ and $\boldsymbol{T}_{s_{k}}^{(j)}$ together to generate the resulting transmit pattern $\boldsymbol{T}^{(l)}$. The packaging scheme is that for ordering pattern $\boldsymbol{T}_{u}^{(i)}$, the ordering state $j$ is incremented by one while $j \leq J$. Inside each $\left(\boldsymbol{T}_{u}^{(i)}, \boldsymbol{T}_{s_{k}}^{(j)}\right)$ th packaging process, in order to put $\boldsymbol{T}_{s_{k}}^{(j)}$ in the right position, we locate the row indices of the nonzero entries in the sparse matrix $\boldsymbol{T}_{u}^{(i)}$. Then, we put the ordering pattern $\boldsymbol{T}_{s_{k}}^{(j)}$ to its corresponding nonzero element in the sparse matrix $\boldsymbol{T}_{u}^{(i)}$ and preserve the original sparse pattern. Taking the combination of $\left(\boldsymbol{T}_{u}^{(2)}, \boldsymbol{T}_{s_{k}}^{(2)}\right)$ for example, the resulting transmit pattern is

$$
\boldsymbol{T}^{(2)}=\left[\begin{array}{ccc}
\mathbf{0} & \mathbf{0} & \boldsymbol{T}_{s_{3}}^{(2)} \\
\mathbf{0} & \boldsymbol{T}_{s_{2}}^{(2)} & \mathbf{0} \\
\boldsymbol{T}_{s_{1}}^{(2)} & \mathbf{0} & \mathbf{0}
\end{array}\right]
$$

For the users equipped with the same number of receive antennas, the total number of ordering states for each user is the same and $\boldsymbol{T}_{s_{1}}^{(j)}=\boldsymbol{T}_{s_{2}}^{(j)}=\boldsymbol{T}_{s_{3}}^{(j)}$. Then, we use $\boldsymbol{T}_{s}^{(j)}$ to denote the ordering patterns for the users and the packaging strategy is simplified by directly implementing the Kronecker product between $\boldsymbol{T}_{u}^{(i)}$ and $\boldsymbol{T}_{s}^{(j)}$

$$
\boldsymbol{T}^{(l)}=\boldsymbol{T}_{u}^{(i)} \otimes \boldsymbol{T}_{s}^{(j)}, 1 \leq l \leq L_{B} .
$$

With the transmit patterns, a list of transmission branches is constructed. Then, a proper selection criterion is developed below to find the branch with the minimum sum of errors among all the branches. The corresponding equivalent channel matrix for a chosen transmit pattern is denoted as $\boldsymbol{H}^{(o)}=\boldsymbol{T}^{(o)} \boldsymbol{H}$. Since we employ the MB strategy to generate extra branches for selection, the BER performance of the proposed MB-THP algorithms will stay the same or have a better performance than the conventional THP algorithms.

The maximum number of branches $L_{B}$ can be equal to $K ! J$ !, however, we restrict the total number of branches to no more than $K \cdot J$ by setting $J=\operatorname{Max}_{k}\left(N_{k}\right)$. Thus, a reasonable system complexity is maintained. It is also not necessary to set $L_{B}$ equal to the maximum number of branches. MB-cTHP and
MB-dTHP can approach the performance with $L_{B}$ branches by using only 2 or 4 branches as will be illustrated in Section V. A total of $L_{B}$ branches is stored at both the transmitter and the receivers, which requires extra memory for storage. A search procedure is also required to select the best pattern for each transmission.

\section{Selection Criterion for the MB-THP}

From the analysis following equations (14) and (15), the multiplication of different transmit patterns $\boldsymbol{T}^{(l)}$ by the row vectors of the channel matrix $\boldsymbol{H}$ results in different error covariance matrices for MB-cTHP and MB-dTHP. For each layer of MB-dTHP, its SNR is inversely proportional to $1 / l_{i, i}^{2}$. For MB-cTHP, it is inversely proportional to $\sum_{i=1}^{S}\left(1 / l_{i, i}^{2}\right)$. Thus, a minimum error selection criterion (MESC) is developed for both MB-cTHP and MB-dTHP to select the best branch according to

$$
l^{(o)}=\arg \min _{1 \leq l \leq L_{B}} \sum_{1 \leq i \leq S}\left(1 / l_{i, i}^{(l)}\right)^{2},
$$

where $l^{(o)}$ is the selected branch. Then, the received signal $\boldsymbol{r}^{(o)}$ is obtained by

$$
\begin{aligned}
\boldsymbol{r}^{(o)^{(\mathrm{dTHP})}} & =\boldsymbol{G}^{(o)}\left(\boldsymbol{H}^{(o)} \boldsymbol{F}^{(o)} \boldsymbol{x}^{(o)}+\boldsymbol{n}\right), \\
\boldsymbol{r}^{(o)^{(\mathrm{TTHP})}} & =\beta\left(\boldsymbol{H}^{(o)} \cdot \frac{1}{\beta} \boldsymbol{F}^{(o)} \boldsymbol{G}^{(o)} \boldsymbol{x}^{(o)}+\boldsymbol{n}\right) .
\end{aligned}
$$

Since the transmit patterns are pre-stored and known both at the transmit and receive terminals, the transmitter can inform the receiver about the index of the selected pattern or the receiver can search for the best pattern. Then, the ordered signal $\boldsymbol{r}^{(o)}$ is transformed back to $\boldsymbol{r}$ by $\boldsymbol{T}^{(o)^{T}}$ at each receive terminal. Next, the transformed signal $r$ is passed through the modulo processing to remove the offset by the perturbation vector $\boldsymbol{d}^{(o)}$, and a quantization function is followed to slice the symbols to the nearest points of the constellation as

$$
\hat{\boldsymbol{s}}=\mathrm{Q}(\mathrm{M}(\boldsymbol{r})),
$$

where $\mathrm{Q}(\cdot)$ is the slicing function and $\mathrm{M}(\cdot)$ is the modulo operation implemented element-wise as in (8). 


\section{E. Derivation of Filters for the MB-MMSE-THP}

It is well-known that MMSE based precoding algorithms always have a better performance than that of ZF based. The filters of the cTHP based MMSE design are deduced from an optimization problem in [19], [20], which results in a high computational complexity since multiple calculations of matrix inverses are required. The orthogonality principle is utilized in [23] to obtain the filters of MMSE-dTHP. In [24], the filters of MMSE-cTHP are derived from an extended system model, which is simpler and more effective compared to the above two methods because the LQ decomposition is utilized. The receive model for MMSE-cTHP based on the extended matrix, however, is not given in [24]. In this work, we derive the filters of the proposed MB-MMSE-cTHP and MB-MMSEdTHP based on the extended matrix and their corresponding receive models are also described.

Define the $N_{r} \times\left(N_{r}+N_{t}\right)$ extended channel matrix $\underline{\boldsymbol{H}}$ for the MB-MMSE precoding schemes as

$$
\underline{\boldsymbol{H}}^{(l)}=\left[\begin{array}{ll}
\boldsymbol{H}^{(l)}, & \sigma_{n} \boldsymbol{I}_{N_{r}}
\end{array}\right],
$$

where $\boldsymbol{H}^{(l)}=\boldsymbol{T}^{(l)} \boldsymbol{H}$. Then, the linear precoding MMSE filter can be rewritten as $\boldsymbol{P}_{\mathrm{MMSE}}^{(l)}=\boldsymbol{A} \underline{\boldsymbol{H}}^{(l)}{ }^{H}\left(\underline{\boldsymbol{H}}^{(l)} \underline{\boldsymbol{H}}^{(l)^{H}}\right)^{-1}$, where $\boldsymbol{A}=\left[\boldsymbol{I}_{N_{t}}, \boldsymbol{0}_{N_{t}, N_{r}}\right]$. By implementing the LQ decomposition of the extended channel matrix $\underline{\boldsymbol{H}}^{(l)}$ we have

$$
\underline{\boldsymbol{H}}^{(l)}=\underline{\boldsymbol{L}}^{(l)} \underline{\boldsymbol{Q}}^{(l)}=\underline{\boldsymbol{L}}^{(l)}\left[\boldsymbol{Q}_{1}^{(l)}, \quad \boldsymbol{Q}_{2}^{(l)}\right],
$$

where $\underline{\boldsymbol{L}}^{(l)}$ is a $N_{r} \times N_{r}$ lower triangular matrix and the $N_{r} \times\left(N_{r}+N_{t}\right)$ matrix $\underline{\boldsymbol{Q}}^{(l)}$ with orthogonal columns can be partitioned into the $N_{r} \times N_{t}$ matrix $\boldsymbol{Q}_{1}^{(l)}$ and the $N_{r} \times N_{r}$ matrix $\boldsymbol{Q}_{2}^{(l)}$. From (29) and (30), the following relations hold

$$
\begin{aligned}
\boldsymbol{H}^{(l)} & =\underline{\boldsymbol{L}}^{(l)} \boldsymbol{Q}_{1}^{(l)}, \\
\underline{\boldsymbol{L}}^{(l)}{ }^{-1} & =\frac{1}{\sigma_{n}} \boldsymbol{Q}_{2}^{(l)}, \\
\boldsymbol{A} \underline{\boldsymbol{Q}}^{(l)} & =\boldsymbol{Q}_{1}^{(l)}{ }^{H} .
\end{aligned}
$$

Therefore, the filters for the MB-MMSE-cTHP and the MBMMSE-dTHP schemes can be obtained as

$$
\begin{aligned}
\boldsymbol{F}^{(l)} & =\underline{\boldsymbol{Q}}^{(l)}{ }^{H}, \\
\boldsymbol{G}^{(l)} & =\operatorname{diag}\left[\underline{l}_{1,1}^{(l)}, \underline{l}_{2,2}^{(l)}, \cdots, \underline{l}_{N_{t}, N_{t}}^{(l)}\right]^{-1}, \\
\boldsymbol{B}^{(l)^{(\mathrm{dTHP})}} & =\boldsymbol{G}^{(l)} \underline{\boldsymbol{L}}^{(l)}, \\
\boldsymbol{B}^{(l)^{(\mathrm{TTHP})}} & =\underline{\boldsymbol{L}}^{(l)} \boldsymbol{G}^{(l)},
\end{aligned}
$$

where $\underline{l}_{i i}^{(l)}$ are the diagonal elements of $\underline{\boldsymbol{L}}^{(l)}$. The received signal for the $l$ th branch is

\begin{tabular}{|c|c|}
\hline Steps & 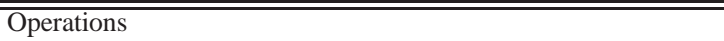 \\
\hline & Compute the extended channel matrix for the $l$ th branch \\
\hline (1) & $\underline{\boldsymbol{H}}^{(l)}=\left[\begin{array}{cc}\boldsymbol{T}^{(l)} \boldsymbol{H}, & \sigma_{n} \boldsymbol{I}_{S}\end{array}\right]$ \\
\hline & Implement the LQ decomposition \\
\hline (2) & $\underline{\boldsymbol{H}}^{(l)}=\underline{\boldsymbol{L}}^{(l)} \boldsymbol{Q}^{(l)}$ \\
\hline & 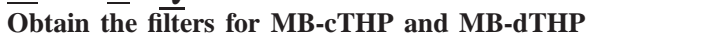 \\
\hline (3) & $\begin{array}{l}\boldsymbol{F}^{(l)}=\underline{\boldsymbol{Q}}^{(l)}{ }^{H}, \boldsymbol{G}^{(l)}=\operatorname{diag}\left[\underline{l}_{1,1}^{(l)}, \underline{l}_{2,2}^{(l)}, \cdots, \underline{l}_{N N_{t}, N_{t}}^{(l)}\right]^{-1}, \\
\left.\boldsymbol{B}^{(l)}{ }^{(\mathrm{cT}} \mathrm{HP}\right) \\
\text { The MESC selection criterion }\end{array}$ \\
\hline (4) & for $\mathrm{j}=1: L_{B} \ddagger \ddagger L_{B}$ is the total number of branches \\
\hline (5) & $\operatorname{MESC}(j)=\sum_{i=1}^{S}\left(1 / l_{i, i}^{2}\right)$ \\
\hline (6) & end \\
\hline (7) & $l^{(o)^{\ddagger \ddagger}}=\operatorname{Min}(\mathrm{MESC}) \ddagger \ddagger l^{(o)}$ is the selected optimal branch \\
\hline (8) & $\begin{array}{l}\text { The successive cancelation process } \\
\text { for } \mathrm{i}=1: S\end{array}$ \\
\hline (9) & $x^{(o)}(i)=s_{i}-\sum_{j \neq i}^{S} b_{i, j} x^{(o)}(j)$ \\
\hline (10) & $x^{(o)}(i)=\mathrm{M}\left(x^{(o)}(i)\right)$ \\
\hline (11) & \\
\hline (12) & $\begin{array}{l}\text { The received signal } \\
\beta=\frac{E\|\boldsymbol{F} \boldsymbol{G} \boldsymbol{x}\|}{\mathrm{E}\|\boldsymbol{s}\|}\end{array}$ \\
\hline (13) & $\boldsymbol{r}^{(o)^{(\mathrm{CTHP})}}=\beta\left(\boldsymbol{H}^{(o)} \cdot \frac{1}{\beta} \boldsymbol{F}^{(o)} \boldsymbol{G}^{(o)} \boldsymbol{x}^{(o)}+\boldsymbol{n}\right)$ \\
\hline (14) & $\boldsymbol{r}^{(o)^{(\mathrm{dTHP})}}=\boldsymbol{G}^{(o)}\left(\boldsymbol{H}^{(o)} \boldsymbol{F}^{(o)} \boldsymbol{x}^{(o)}+\boldsymbol{n}\right)$ \\
\hline (15) & $\hat{\boldsymbol{s}}^{(\mathrm{cTHP})}=\mathrm{Q}\left(\mathrm{M}\left(\boldsymbol{T}^{(o)^{T}} \boldsymbol{r}^{(o)^{(\mathrm{cTHP})}}\right)\right)$ \\
\hline (16) & $\hat{\boldsymbol{s}}^{(\mathrm{dTHP})}=\mathrm{Q}\left(\mathrm{M}\left(\boldsymbol{T}^{(o)^{T}} \boldsymbol{r}^{(o)^{(\mathrm{dTHP})}}\right)\right)$ \\
\hline
\end{tabular}

$$
\begin{aligned}
& \boldsymbol{r}^{(l)^{(\mathrm{dTHP})}}=\boldsymbol{G}^{(l)}\left(\boldsymbol{H}^{(l)} \boldsymbol{A} \boldsymbol{F}^{(l)} \boldsymbol{x}^{(l)}+\boldsymbol{n}\right), \\
& \boldsymbol{r}^{(l)^{(\mathrm{CTHP})}}=\beta\left(\boldsymbol{H}^{(l)} \cdot \frac{1}{\beta} \boldsymbol{A} \boldsymbol{F}^{(l)} \boldsymbol{G}^{(l)} \boldsymbol{x}^{(l)}+\boldsymbol{n}\right) .
\end{aligned}
$$

It is worth noting that the multiplication by $\boldsymbol{A}$ will not result in transmit power amplification since $\boldsymbol{A} \boldsymbol{A}^{H}=\boldsymbol{I}_{N_{t}}(\boldsymbol{A}$ is pseudounitary). The implementation steps of the MB-MMSE-THP algorithms are summarized in Table I.
TABLE I: Proposed MB-MMSE-THP Algorithms

\section{Performance Analysis}

In this section, we consider a performance analysis in terms of error covariance, sum-rate, and computational complexity.

\section{A. Performance Analysis of the Error Covariance Matrix}

The autocorrelation matrices of the interference-plus-noise power in ZF-dTHP and ZF-cTHP have been given in [30], however, the comparison has not been done. In this section, we illustrate the BER performances in terms of error covariance. For the comparison between ZF-dTHP and ZF-cTHP, we assume $i$ is an arbitrary layer, then from equations (14) and (15) we have

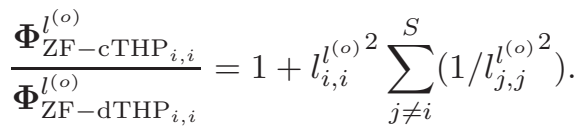

That is, $\forall i: \boldsymbol{\Phi}_{\mathrm{ZF}-\mathrm{dTHP}_{i, i}}^{l^{(o)}}<\boldsymbol{\Phi}_{\mathrm{ZF}-\mathrm{cTHP}_{i, i}}^{l^{(o)}}$. Since the BER performance is largely related to the error covariance matrix, we expect a better BER performance achieved by ZF-dTHP over ZF-cTHP. This is also verified by the simulation result in [30], from which a slightly better BER performance of ZFdTHP over ZF-cTHP is reported.

The comparison between MMSE-dTHP and MMSE-cTHP, however, has not been analyzed nor simulated in the literature so far. Substituting (31), (33), (34) and (36) into (38), we can get the error covariance matrix for MMSE-dTHP as

$$
\boldsymbol{\Phi}_{\text {MMSE-dTHP }}^{(l)}=\operatorname{diag}\left(\sigma_{n} / \underline{l}_{1,1}^{(l)}, \cdots, \sigma_{n} / \underline{l}_{S, S}^{(l)}\right)^{2} .
$$

For the MMSE-cTHP we start from the calculation of $\beta$ for a more accurate expression by

$$
\beta^{2}=\frac{\mathrm{E}\left\|\boldsymbol{A} \boldsymbol{F}^{(l)} \boldsymbol{G}^{(l)} \boldsymbol{x}^{(l)}\right\|^{2}}{\sigma_{s}^{2}},
$$


where $\sigma_{s}^{2}=\mathrm{E}\|\boldsymbol{s}\|^{2}$. Since $\boldsymbol{x}^{(l)}=\boldsymbol{B}^{(l)}{ }^{-1} \boldsymbol{v}^{(l)}, \boldsymbol{B}^{(l)}=$ $\underline{\boldsymbol{L}}^{(l)} \boldsymbol{G}^{(l)}$ and $\underline{\boldsymbol{L}}^{(l)-1}=\frac{1}{\sigma_{n}} \boldsymbol{Q}_{2}^{(l)}$, the multiplication $\boldsymbol{A} \boldsymbol{F}^{(l)} \boldsymbol{G}^{(l)} \boldsymbol{x}^{(l)}$ is obtained as

$$
\boldsymbol{A F}^{(l)} \boldsymbol{G}^{(l)} \boldsymbol{x}^{(l)}=\frac{1}{\sigma_{n}} \boldsymbol{A} \boldsymbol{F}^{(l)} \boldsymbol{Q}_{2}^{(l)} \boldsymbol{v}^{(l)} .
$$

Then, by applying the equivalence $\operatorname{tr}(\boldsymbol{A} \boldsymbol{B} \boldsymbol{C})=\operatorname{tr}(\boldsymbol{C} \boldsymbol{A} \boldsymbol{B})$, the normalization factor $\beta$ can be expressed as

$$
\beta^{2}=\frac{\sigma_{v}^{(l)}}{\sigma_{n}^{2} \sigma_{s}^{2}}
$$

where the quantity $\sigma_{v}^{(l)}{ }^{2}$ is the variance of $\boldsymbol{v}^{(l)}$. Therefore, the error covariance matrix for MMSE-cTHP is obtained as

$$
\boldsymbol{\Phi}_{\mathrm{MMSE}-\mathrm{cTHP}}^{(l)}=\operatorname{diag}\left(\frac{\sigma_{v}^{(l)^{2}}}{\sigma_{s}^{2}}, \cdots, \frac{\sigma_{v}^{(l)^{2}}}{\sigma_{s}^{2}}\right) .
$$

By changing the transmit signal order, different perturbation vectors $\boldsymbol{d}^{(l)}$ are obtained in MB-MMSE-cTHP. The multibranch processing is actually used to select the one with the minimum $\sigma_{v}^{(l)^{2}}$ among all the $L_{B}$ branches in MB-MMSEcTHP algorithms.

For the comparison between MB-MMSE-dTHP and MMSE-dTHP, we have the proposition below.

Proposition 1: The trace of the error covariance matrix for the proposed MB-MMSE-dTHP technique is upper bounded by that of the conventional MMSE-dTHP scheme, i.e.,

$$
\operatorname{tr}\left(\boldsymbol{\Phi}_{\mathrm{MB}-\mathrm{MMSE}-\mathrm{dTHP}}\right) \leq \operatorname{tr}\left(\boldsymbol{\Phi}_{\mathrm{MMSE}-\mathrm{dTHP}}\right)
$$

Proof: From the MESC selection criterion in (25), the selected branch $l^{(o)}$ corresponds to the sum of the elements associated with the smallest value, i.e.,

$$
\operatorname{tr}\left(\boldsymbol{\Phi}_{\mathrm{MB}-\mathrm{MMSE}-\mathrm{dTHP}}\right)=\sum_{1 \leq i \leq S}\left(1 / \underline{l}_{i, i}^{(o)}\right)^{2} .
$$

With the MESC selection criterion, we have

$$
\sum_{1 \leq i \leq S}\left(1 / \underline{l}_{i, i}^{(o)}\right)^{2} \leq \sum_{1 \leq i \leq S}\left(1 / \underline{l}_{i, i}^{(l)}\right)^{2}, l=1,2, \cdots, L_{B} .
$$

By writing the above quantities without the sum, we get

$$
\begin{gathered}
\left(\frac{1}{\underline{l}_{1,1}^{(o)}}\right)^{2}+\cdots+\left(\frac{1}{\underline{l}_{S, S}^{(o)}}\right)^{2} \leq\left(\frac{1}{\underline{l}_{1,1}^{(l)}}\right)^{2}+\cdots+\left(\frac{1}{\underline{l}_{S, S}^{(l)}}\right)^{2}, \\
{\left[\left(\frac{1}{\underline{l}_{1,1}^{(o)}}\right)^{2}-\left(\frac{1}{\underline{l}_{1,1}^{(l)}}\right)^{2}\right]+\cdots+\left[\left(\frac{1}{\underline{l}_{S, S}^{(o)}}\right)^{2}-\left(\frac{1}{\underline{l}_{S, S}^{(l)}}\right)^{2}\right] \leq 0 .}
\end{gathered}
$$

If we choose $\underline{l}_{i, i}^{(o)}$ to be identical to $\underline{l}_{i, i}^{(l)}$ then we prove the equality $\operatorname{tr}\left(\boldsymbol{\Phi}_{\mathrm{MB}-\mathrm{MMSE}-\mathrm{dTHP}}\right)=\operatorname{tr}\left(\boldsymbol{\Phi}_{\mathrm{MMSE}-\mathrm{dTHP}}\right)$. If we choose at least one element $\underline{l}_{i, i}^{(o)}>\underline{l}_{i, i}^{(l)}$ or $\underline{l}_{i, i}^{(o)}-\underline{l}_{i, i}^{(l)}=\epsilon$ while keeping the others identical $\underline{l}_{j, j}^{(o)}=\underline{l}_{j, j}^{(l)}, j \neq i$ then we prove the inequality $\operatorname{tr}\left(\boldsymbol{\Phi}_{\mathrm{MB}-\mathrm{MMSE}-\mathrm{dTHP}}\right)<\operatorname{tr}\left(\boldsymbol{\Phi}_{\mathrm{MMSE}-\mathrm{dTHP}}\right)$, where $\epsilon$ is a small real positive value
For MMSE-cTHP, the overall SNR performance is influenced by the sum of each layer, then from (45) we have

$$
\begin{aligned}
\operatorname{tr}\left(\boldsymbol{\Phi}_{\text {MMSE-cTHP }}\right) & =\frac{K \sigma_{v}^{(l)^{2}}}{\sigma_{s}^{2}}, \\
\operatorname{tr}\left(\boldsymbol{\Phi}_{\mathrm{MB}-\mathrm{MMSE}-\mathrm{cTHP}}\right) & =\frac{K \sigma_{v}^{(o)^{2}}}{\sigma_{s}^{2}} .
\end{aligned}
$$

Because of the MESC selection process, we have obtained that $\forall l: \sigma_{v}^{(o)^{2}} \leq \sigma_{v}^{(l)^{2}}$. Thus, it is straightforward to conclude that

$$
\operatorname{tr}\left(\boldsymbol{\Phi}_{\text {MB-MMSE-cTHP }}\right) \leq \operatorname{tr}\left(\boldsymbol{\Phi}_{\text {MMSE-cTHP }}\right) .
$$

Therefore, we expect that a better BER performance can be achieved by the proposed MB-dTHP and MB-cTHP, respectively, as compared to their original counterparts.

\section{B. Sum-Rate Performance Analysis}

From the analysis illustrated in Section III, the MU-MIMO channel is decomposed into parallel AWGN channels in the THP systems. Therefore, the $i$ th SNR for the $l$ th branch transmit signal of MB-ZF-THP is given by [2], [30]

$$
\begin{aligned}
\gamma_{i}{ }^{(l)}{ }^{(\mathrm{MB}-\mathrm{ZF}-\mathrm{dTHP})} & =\frac{\sigma_{s}^{2}}{\sigma_{n}^{2}\left(1 / l_{i, i}^{(l)^{2}}\right)}, \\
\gamma_{i}^{(l)}{ }^{(\mathrm{MB}-\mathrm{ZF}-\mathrm{cTHP})} & =\frac{\sigma_{s}^{2}}{\sigma_{n}^{2} \sum_{i=1}^{S}\left(1 / l_{i, i}^{(l)}\right)} .
\end{aligned}
$$

Then, the achievable sum rates for the $l$ th branch of MB-ZFdTHP and MB-ZF-cTHP are respectively given by

$$
\begin{aligned}
C_{(\mathrm{MB}-\mathrm{ZF}-\mathrm{dTHP})}^{(l)} & =\sum_{i=1}^{S} \log \left(1+\frac{\sigma_{s}^{2} l_{i, i}^{(l)^{2}}}{\sigma_{n}^{2}}\right) \\
C_{(\mathrm{MB}-\mathrm{ZF}-\mathrm{cTHP})}^{(l)} & =S \log \left(1+\frac{\sigma_{s}^{2}}{\sigma_{n}^{2} \sum_{i=1}^{S}\left(1 / l_{i, i}^{(l)}\right)^{2}}\right)
\end{aligned}
$$

From equations (41) and (42), the achievable sum rates of MB-MMSE-dTHP and MB-MMSE-cTHP can be expressed, respectively, as follows

$$
\begin{aligned}
& C_{(\mathrm{MB}-\mathrm{MMSE}-\mathrm{dTHP})}^{(l)}=\sum_{i=1}^{S} \log \left(1+\frac{\sigma_{s}^{2} l_{i, i}^{(l)}}{\sigma_{n}^{2}}\right), \\
& C_{(\mathrm{MB}-\mathrm{MMSE}-\mathrm{cTHP})}^{(l)}=S \log \left(1+\frac{\sigma_{s}^{4}}{\sigma_{v}^{(l)}}\right) .
\end{aligned}
$$

From (54) and (56), the difference of the overall average SNR for the $l$ th branch is small. Thus, we expect that MBMMSE-dTHP with different branches shares a similar sumrate performance. For MB-MMSE-cTHP, the $\sigma_{v}^{(o)^{2}}$ of the selected $l^{(o)}$ th branch has the minimum value among all the branches because of the multi-branch processing and the selection, that is

$$
\sigma_{v}^{(o)^{2}} \leq \sigma_{v}^{(l)^{2}}, l=1, \cdots, L_{B},
$$

Thus, we have

$$
C_{(\mathrm{MMSE}-\mathrm{cTHP})} \leq C_{(\mathrm{MB}-\mathrm{MMSE}-\mathrm{cTHP})}^{(o)},
$$

which means the sum-rate performance of MMSE-cTHP can be improved by the proposed MB-MMSE-cTHP algorithm. 
TABLE II: Comparison of the complexity

\begin{tabular}{|c|c|c|}
\hline Algorithm & FLOPS & Case \\
\hline$\overline{\mathrm{ZF}}$ & $16 n^{3}+3 n^{2}-2 n$ & $\overline{3552}$ \\
\hline MMSE & $16 n^{3}+3 n^{2}$ & 3564 \\
\hline $\mathrm{BD}$ & $\begin{array}{l}K\left(72 N_{k}^{3}+72 n_{i}^{2} n+32 N_{k} n^{2}\right. \\
\left.-2 N_{k}^{2}+32 n \bar{N}_{k}^{2}+64 \bar{N}_{k}^{3}\right)\end{array}$ & 35304 \\
\hline RBD & $\begin{array}{l}K\left(72 N_{k}^{3}+72 N_{k}^{2} n+32 N_{k} n^{2}-2 N_{k}^{2}\right. \\
\left.+8 n^{3}+18 n+\bar{n}_{i}+32 n \bar{N}_{k}^{2}+64 \bar{N}_{k}^{3}\right)\end{array}$ & 40824 \\
\hline ZF-THP & $\frac{40}{3} n^{3}+10 n^{2}+22 n$ & 3372 \\
\hline MMSE-THP [19] & $24 n^{4}+48 n^{3}+n^{2}$ & 41508 \\
\hline MMSE-THP & $\frac{64}{3} n^{3}+10 n^{2}+22 n$ & 5100 \\
\hline MB-ZF-THP & $L_{B}\left(\frac{40}{3} n^{3}+10 n^{2}+22 n\right)$ & 6744 \\
\hline MB-MMSE-THP & $L_{B}\left(\frac{64}{3} n^{3}+10 n^{2}+22 n\right)$ & 10200 \\
\hline ZF-VP/MMSE-VP & $\begin{array}{l}8 \sum_{k=1}^{n} \frac{M k \pi \frac{k}{2}}{\Gamma(k / 2+1)} d^{k} \\
\quad+16 n^{2}-2 n+4\end{array}$ & $4.8 \cdot 10^{7}$ \\
\hline
\end{tabular}

\section{Complexity Analysis}

In this section we use the total number of floating point operations (FLOPs) to measure the computational complexity of the proposed and existing algorithms. The number of FLOPs for the LQ decomposition is obtained by assuming that the LQ decomposition is computed by using the Householder transformation given in [38]. We summarize the total number of FLOPs needed for the matrix operations below:

- Multiplication of $m \times n$ and $n \times p$ complex matrices: $8 m n p-2 m p$

- LQ decomposition of an $m \times n(m \leq n)$ complex matrix: $8 m^{2}\left(n-\frac{1}{3} m\right)$

- Pseudo-inversion of an $m \times n$ complex matrix: $\left(\frac{4}{3} m^{3}+\right.$ $\left.7 m^{2} n-m^{2}-2 m n\right)$.

The number of FLOPs needed for BD and RBD can be found in [39], [40]. The computational complexity of MMSETHP based on multiple matrix inversions in [19] has been given in [41]. The complexity reported in [41], however, is only computed in terms of the number of multiplications and additions. For the complex multiplications and additions, it respectively needs 6 and 2 FLOPs. Thus, the number of FLOPs needed by MMSE-THP in [19] is at least $24 N_{r}^{4}+48 N_{r}^{3}+$ $N_{t} N_{r}$. For MMSE-THP based on the Cholesky factorization in [41], the number of FLOPs needed is at least $\frac{20}{3} N_{r}^{3}+8 N_{r}^{2} N_{t}$. The ZF-VP in [9] and MMSE-VP in [42] are implemented by using the sphere decoder (SD) algorithm which is employed for sphere encoding. The complexity of SD is associated with the constellation size $M$ and the radius $d$ which is chosen to be a scaled version of the noise variance [43]. The required multiplications and additions of SD are given in [44].

For simplicity, we assume that the number of transmit antennas $N_{t}$ and the number of receive antennas $N_{r}$ are equal to $n$. From the above derivation, MB-MMSE-dTHP and MB-MMSE-cTHP share the same computational complexity. The number of FLOPs for the above precoding algorithms are listed in Table II, where $\bar{N}_{k}=n-N_{k}$. In case of system dimension $n=6$, number of users $K=3$, each user equipped with $N_{k}=2$ receive antennas and number of branches $L_{B}=2$, the required number of FLOPs of MB-ZFTHP and MB-MMSE-THP is much lower than the BD, RBD, conventional MMSE-THP in [19] and VP algorithms.
The required number of FLOPs of the proposed and existing algorithms is simulated for different system dimensions and the results are depicted in Fig. 3. It is clear that VP shows the highest complexity. The computational cost of $\mathrm{BD}, \mathrm{RBD}$, and MMSE-THP in [19] is relatively high compared to the proposed MB-MMSE-THP algorithms due to multiple SVD or matrix inversion operations are implemented. Moreover, the proposed MB-MMSE-THP algorithm with $L_{B}=2$ and $L_{B}=$ 4 branches has a complexity that is slightly higher than the ZF-THP, MMSE, and MMSE-THP algorithms especially when the system dimension is below 10 .

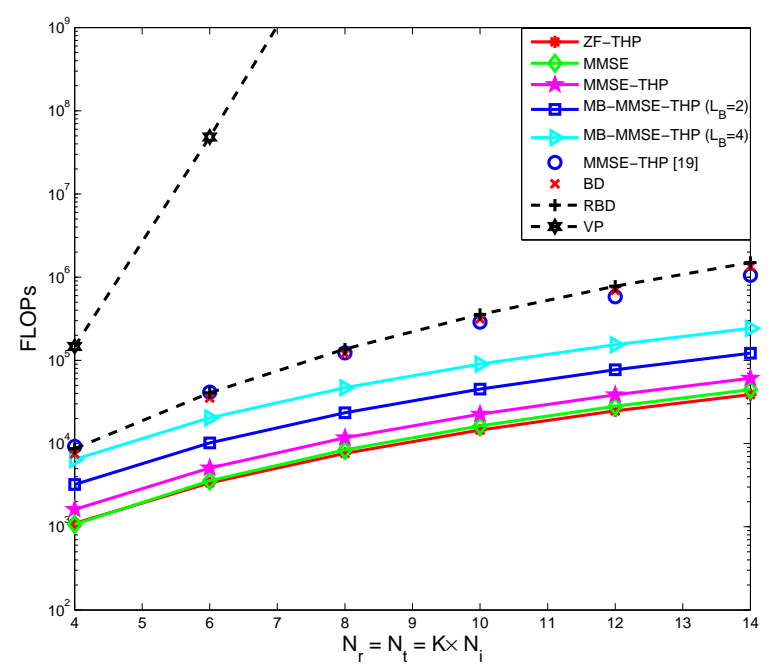

Fig. 3: Complexity Analysis (The proposed MB-cTHP and MB-dTHP share the same complexity).

\section{Simulation Results}

In this section, we assess the performance of the proposed MB-THP algorithms. A system with $N_{t}=8$ transmit antennas and $K=4$ users each equipped with $N_{k}=2$ receive antennas is considered; this scenario is denoted as the $(2,2,2,2) \times 8$ case. The quantity $E_{b} / N_{0}$ is defined as $E_{b} / N_{0}=\frac{N_{r} E_{s}}{N_{t} N \sigma_{n}^{2}}$ with $N$ being the number of information bits transmitted per channel symbol. Uncoded QPSK and 16-QAM modulation schemes are employed in the simulations. The channel matrix $\boldsymbol{H}$ is assumed to be a complex i.i.d. Gaussian matrix with zero mean and unit variance. The number of branches employed for MB-MMSE-THP is $L_{B}=2,4,6,8$, respectively. The number of simulation trials is $10^{6}$ and the packet length is $10^{2}$ symbols.

\section{A. Perfect Channel State Information Scenario}

As illustrated in Fig. 4a the BER performance of the $\mathrm{BD}$ and $\mathrm{RBD}$ precoding algorithms is worse than that of the THP algorithms. For the THP algorithms, a better BER performance is offered by ZF-dTHP over ZF-cTHP since $\forall i: \boldsymbol{\Phi}_{\mathrm{ZF}-\mathrm{dTHP}_{i, i}}^{l^{(o)}}<\boldsymbol{\Phi}_{\mathrm{ZF}-\mathrm{cTHP}}^{l_{i, i}^{(o)}}$ as we illustrated in (40). However, a much better BER performance is achieved by MMSE-cTHP than MMSE-dTHP, which verifies the analysis developed in Section IV. The comparison among nonlinear 


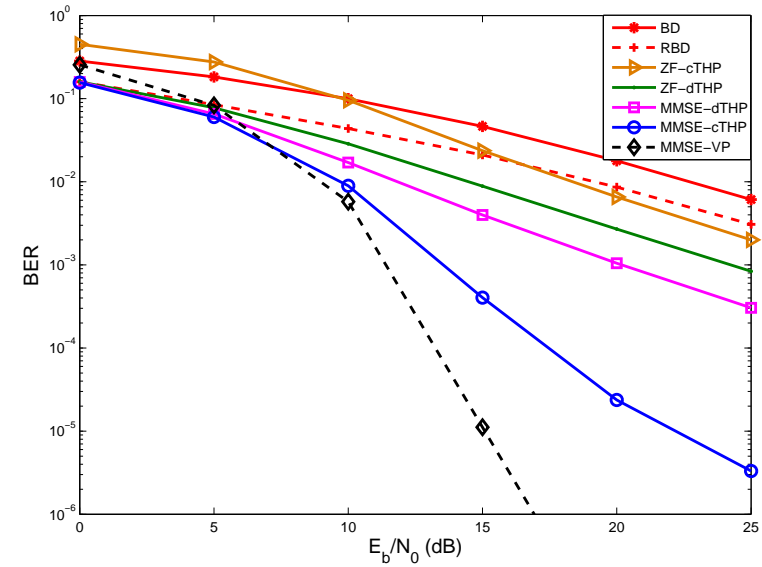

(a) BER performance of THP, QPSK

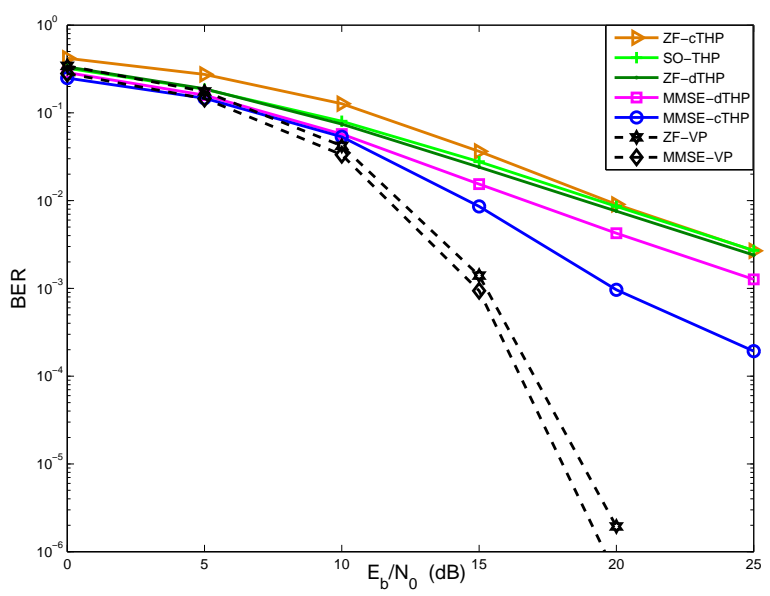

(b) BER performance of THP, 16-QAM

Fig. 4: BER performance of THP

precoding algorithms with 16-QAM is displayed in Fig. 4b The same phenomenon is also observed for the two types of THP with 16-QAM. A slightly better BER performance is offered by ZF-dTHP over ZF-cTHP, whereas, the situation is reversed for MMSE-THP. The THP with successive BD implementation (SO-THP) algorithm in [28] shows a slightly better performance than ZF-cTHP at low $E_{b} / N_{0}$ s, however, its performance is almost the same as ZF-dTHP and ZF-cTHP at high $E_{b} / N_{0} \mathrm{~s}$. The maximum transmit diversity order is achieved by ZF-VP and MMSE-VP algorithms.

The BER performance of the proposed MB-MMSE-cTHP with 16-QAM and QPSK are shown in Fig. 5a and Fig. 5b respectively. From Fig. 5a, the proposed MB-MMSE-cTHP with $L_{B}=2,4,8$ branches has a gain of more than $2 \mathrm{~dB}$, $3 \mathrm{~dB}$, and $3.4 \mathrm{~dB}$ as compared to the conventional MMSEcTHP and the performance gap between MB-MMSE-cTHP with $L_{B}=4$ and MMSE-VP is only $2 \mathrm{~dB}$ at the BER of $10^{-3}$. For the QPSK modulation in Fig. 5b the BER performance of MB-MMSE-cTHP with $L_{B}=4$ is better than MMSE-VP at low $E_{b} / N_{0} \mathrm{~S}$ and is very close to that of MMSE-VP at the BER of $10^{-3}$ but requires a much lower computational complexity.

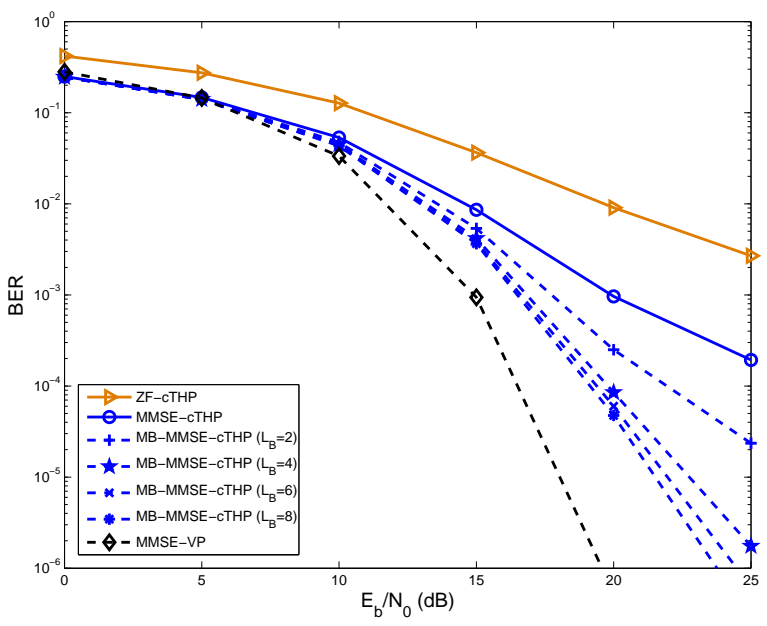

(a) BER performance of cTHP, $(2,2,2,2) \times 8$ MIMO, 16-QAM.

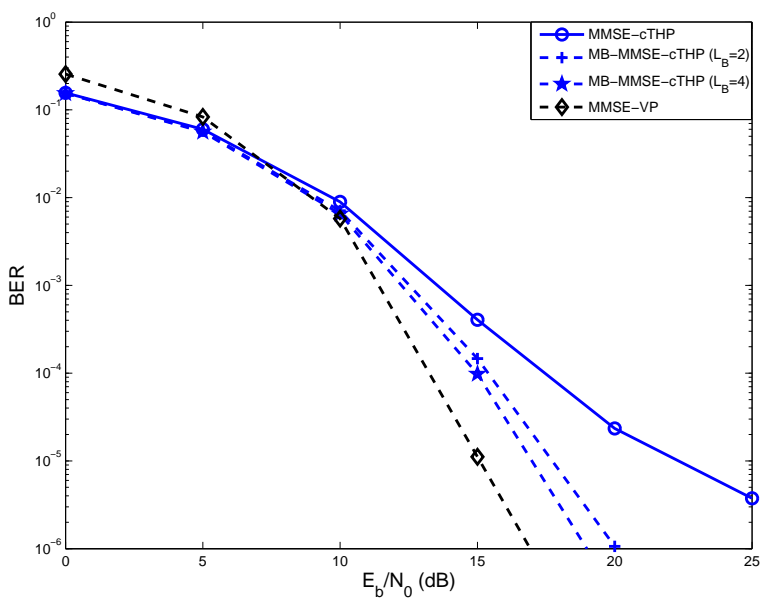

(b) BER performance of cTHP, $(2,2,2,2) \times 8$ MIMO, QPSK.

Fig. 5: BER performance of cTHP

Fig. 6 displays the BER performance of the proposed MBMMSE-dTHP algorithms. For the proposed MB-MMSE-dTHP with $L=2,4,8$ branches, there is a gain of more than $3.6 \mathrm{~dB}$, $6 \mathrm{~dB}$, and $7 \mathrm{~dB}$ as compared to the conventional MMSE-dTHP at the BER of $10^{-3}$, respectively.

As illustrated by Fig. 5 and Fig. 6, the transmit diversity of the proposed MB-MMSE-cTHP and MB-MMSE-dTHP algorithms is between the VP and the conventional MMSETHP algorithms because a list of branches is constructed and the best candidate is selected by the proposed algorithms. It is worth noting that for both MB-MMSE-cTHP and MBMMSE-dTHP with only 2 branches, there is a considerable performance improvement and their BER performances with 4 branches can approach the one with 8 branches. Especially for MB-MMSE-cTHP, its BER performance with only 4 branches is not far from MMSE-VP with much less computational complexity.

Fig. $7 \mathrm{a}$ and Fig. $7 \mathrm{~b}$ display the sum-rate performance of the proposed MB-MMSE-cTHP and MB-MMSE-dTHP algorithms, respectively. From Fig. 7a, we can find that the sum 


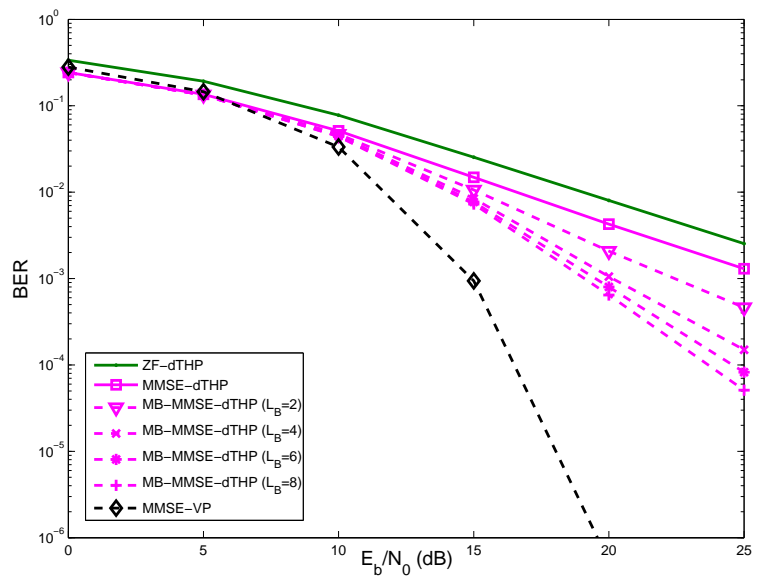

Fig. 6: BER performance of dTHP, $(2,2,2,2) \times 8$ MIMO, 16-QAM.

rates of MB-MMSE-cTHP is improved with the increase of $L_{B}$ as we revealed in (59). When $L_{B}$ is increased to 4 , it can achieve almost the same sum-rate performance as with 8 branches. The SO-THP in [28] has shown a better sumrate performance than MB-MMSE-cTHP algorithms for high values of $E_{b} / N_{0}$. For MB-MMSE-dTHP, however, they share almost the same sum-rate performance with different branches. This phenomenon confirms the analysis developed in Section IV. Another interesting phenomenon can be observed by comparing these two figures is that the sum-rate performance of MB-MMSE-cTHP is better than MB-MMSE-dTHP at low values of $E_{b} / N_{0}$, while MB-MMSE-dTHP offers a very good performance at high values of $E_{b} / N_{0}$.

\section{B. Correlated Channel State Information Scenario}

Here, we study the impact of correlated channels on the performance of the proposed and existing algorithms. A correlated channel matrix can be obtained using the Kronecker model [45]

$$
\boldsymbol{H}_{c}=\boldsymbol{R}_{r}^{\frac{1}{2}} \boldsymbol{H} \boldsymbol{R}_{t}^{\frac{1}{2}} .
$$

For the case of an urban wireless environment, the UE is always surrounded by rich scattering objects and the channel is most likely to be modeled by an independent Rayleigh fading channel at the receive side [46]. Hence, we assume $\boldsymbol{R}_{r}=\boldsymbol{I}_{N_{r}}$, and we have

$$
\boldsymbol{H}_{c}=\boldsymbol{H} \boldsymbol{R}_{t}^{\frac{1}{2}} .
$$

To study the effect of antenna correlations, random realizations of correlated channels are generated according to the exponential correlation model [47] such that the elements of $\boldsymbol{R}_{t}$ are given by

$$
r_{i, j}=\left\{\begin{array}{ll}
r^{j-i}, & i \leq j \\
r_{j, i}^{*}, & i>j
\end{array},|r| \leq 1,\right.
$$

where $r$ is the correlation coefficient between any two neighboring antennas. This correlation model is suitable for our study since, in practice, the correlation between neighboring

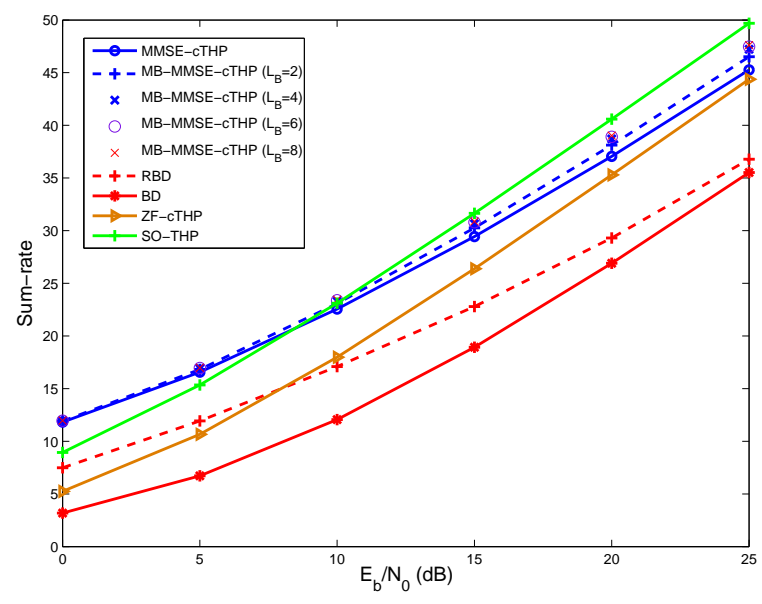

(a) Sum-rate performance of cTHP, $(2,2,2,2) \times 8$ MIMO, 16-QAM.

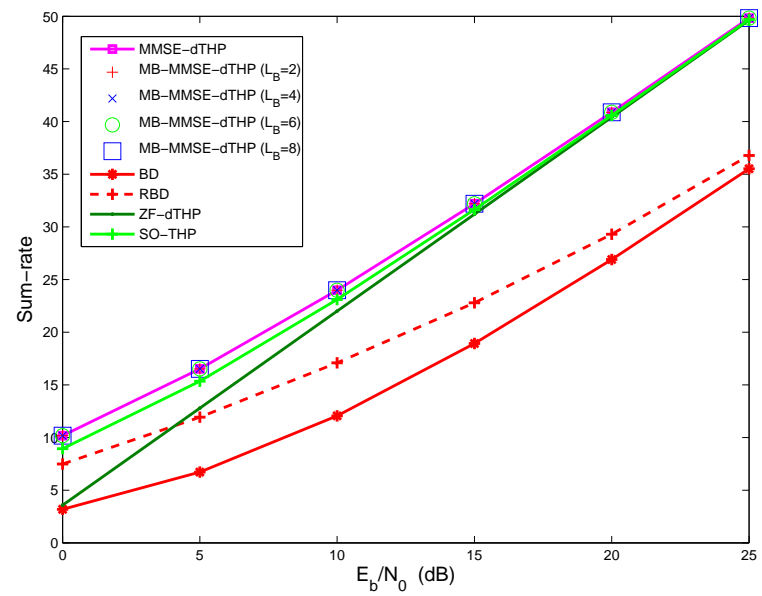

(b) Sum-rate performance of dTHP, $(2,2,2,2) \times 8$ MIMO, 16-QAM.

Fig. 7: Sum-rate performance of THP.

channels is higher than that between distant channels. In the following Fig. 8, we examine the performance of the proposed MB-MMSE-THP algorithms with $|r|=0.5$. The simulation results show that with the spatial correlation, the proposed MB-MMSE-THP algorithms still offer a better performance compared to their conventional counterparts and the MMSEcTHP is more sensitive to the spatial correlation.

\section{The impact of imperfect channels}

For the precoding techniques to work, CSI is required at the transmit side. This is natural for time-division duplex (TDD) systems because the uplink and downlink share the same frequency band. For frequency-division duplex (FDD) systems, however, the CSI needs to be estimated at the receiver and fed back to the transmitter. Assuming perfect CSI is impractical due to the often inaccurate channel estimation and the CSI feedback errors, we need to evaluate the impact of imperfect CSI on the performance of precoders. The channel errors can be modeled as a complex random Gaussian noise matrix $\boldsymbol{E}$ with i.i.d. entries of zero mean and variance $\sigma_{e}^{2}$. The 


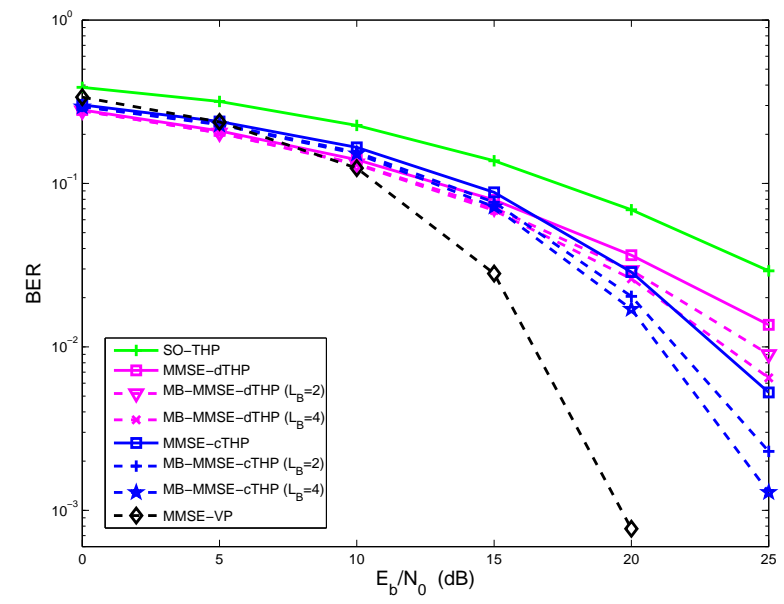

(a) BER with spatial correlation, $(2,2,2,2) \times 8$ MIMO, 16-QAM.

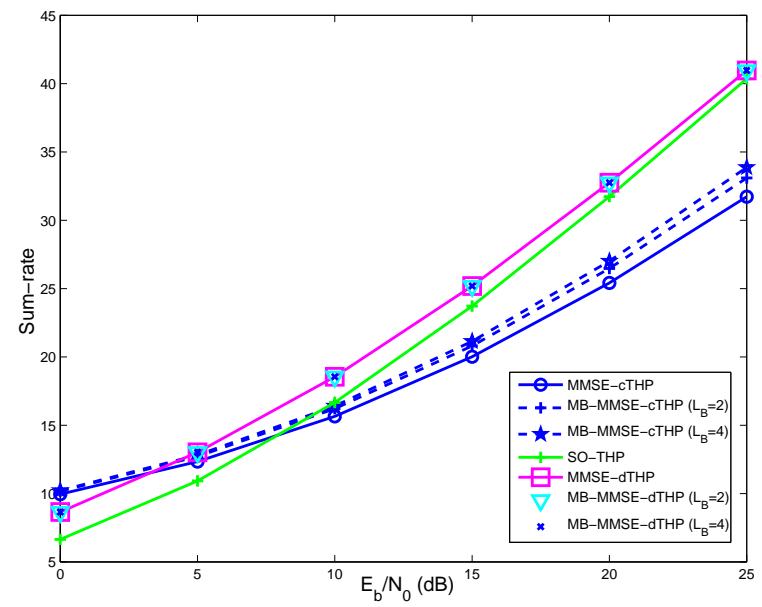

(b) Sum-rate with spatial correlation, $(2,2,2,2) \times 8$ MIMO, 16-QAM.

Fig. 8: Performance with correlated channel.

imperfect channel matrix $\boldsymbol{H}_{e}$ is defined as [3]

$$
\boldsymbol{H}_{e}=\boldsymbol{H}+\boldsymbol{E} .
$$

Fig. 9 illustrates the BER performance of the above precoding algorithms with imperfect CSI at $E_{b} / N_{0}=20 \mathrm{~dB}$. The BER performance gets worse for all the precoding algorithms with the increase of $\sigma_{e}^{2}$. The performance advantage of the proposed MB-MMSE-THP algorithms are not changed at low values of $\sigma_{e}^{2}$, while it degrades faster for higher values of $\sigma_{e}^{2}$ due to the nonlinearity of the algorithms.

It is worth noting that MMSE-cTHP loses its BER performance advantage to MMSE-dTHP for channel errors with a variance larger than $\sigma_{e}^{2}=0.14$ as shown in Fig. 9 which illustrates that the cTHP structure is more sensitive than the dTHP structure to imperfect channels. Therefore, more feedback bits are needed by cTHP than dTHP in realistic systems. A robust optimization of THP based on the meansquared-error (MSE) has been developed in [33] to alleviate the impact of CSI errors. We leave a robust optimization under the MB-cTHP and MB-dTHP framework as a future extension.
Although less feedback information is required for dTHP in practice, the corresponding scaling matrix needs to be transmitted to each distributed receiver, which requires an extra control overhead or additional feedforward information. Since the feedback issue is not the main focus of this work, we leave it for further research.

For MB-MMSE-cTHP and MB-MMSE-dTHP, we have found that

- a better BER performance is obtained by MB-MMSEcTHP compared to MB-MMSE-dTHP.

- MB-MMSE-dTHP can lead to higher system sum rates and more flexible sub-channel management. The sum-rate performance of MB-MMSE-cTHP is not as good as MBMMSE-dTHP but it can be improved with an increase of the number of branches.

- MB-MMSE-cTHP is more sensitive to imperfect CSI than MB-MMSE-dTHP but a simplified receiver structure for MB-MMSE-cTHP has been achieved due to the fact that the decoding matrix $\boldsymbol{G}_{k}$ is not required at each receiver in MB-MMSE-cTHP.

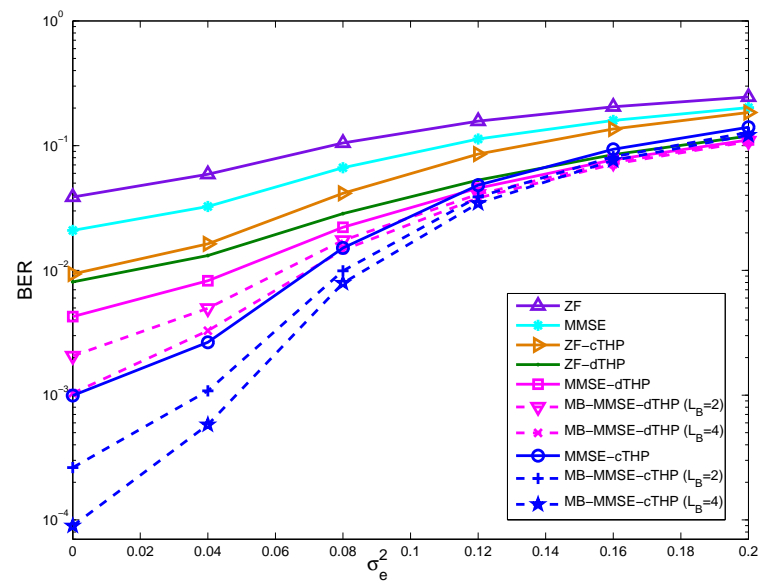

Fig. 9: BER as a function of the variance of CSI error $\sigma_{e}^{2}$ for $E_{b} / N_{0}=$ $20 \mathrm{~dB},(2,2,2,2) \times 8$ MIMO, 16-QAM.

\section{CONCLUSIONS}

In this paper, MB-MMSE-cTHP and MB-MMSE-dTHP algorithms have been proposed for MU-MIMO systems with multiple receive antennas. The proposed MB-MMSE-THP algorithms exploit the degrees of freedom for transmission by constructing a list of branches, which results in extra transmit diversity gains. Moreover, the required computational complexity is still reasonable since the filters of MB-MMSETHP are derived based on an LQ decomposition. A comprehensive performance analysis has been carried out and a wide range of comparisons have been conducted with existing precoding algorithms, including the $\mathrm{BD}, \mathrm{RBD}$, THP, SOTHP, VP algorithms. Simulation results have illustrated that a considerable improvement is achieved with only 2 or 4 branches, which reveals the value of the proposed MB-MMSETHP algorithms for practical applications. Since a set of 
parallel channels is obtained with the application of the MBTHP algorithms, power loading schemes can be employed to optimize the power used over the channels.

\section{REFERENCES}

[1] L. Liu, R. Chen, S. Geirhofer, K. Sayana, Z. Shi and Y. Zhou, "Downlink MIMO in LTE-Advanced: SU-MIMO vs. MU-MIMO," IEEE Commun. Mag., vol. 50, issue: 2, pp. 140-147, Feb. 2012.

[2] D. Tse and P. Viswanath, Fundamentals of wireless communications. Cambridge University Press, 2005

[3] C. Windpassinger, "Detection and precoding for multiple input multiple output channels," Ph.D. dissertation, University Erlangen-Nurnberg, Germany, 2004.

[4] C. Peel, B. M. Hochwald and A. Swindlehurst, "A vector-perturbation technique for near capacity multiantenna multiuser communication - Part I: channel inversion and regularization," IEEE Trans. Commun., vol. 52, no. 1, pp. 195-202, Jan. 2005.

[5] M. Joham, W. Utschick and J. Nossek, "Linear transmit processing in MIMO communications systems," IEEE Trans. Signal Process., vol. 53 no. 8, pp. 2700-2712, Aug. 2005

[6] Q. Spencer, A. Swindlehurst and M. Haardt, "Zero-forcing methods for downlink spatial multiplexing in multiuser MIMO channels," IEEE Trans. Signal Process., vol. 52, no. 2, pp. 461-471, Feb. 2004.

[7] L. Choi and R. Murch, "A transmit preprocessing technique for multiuser MIMO systems using a decomposition approach," IEEE Trans. Wireless Commun., vol. 3, no. 1, pp. 20-24, Jan. 2004.

[8] V. Stankovic and M. Haardt, "Generalized design of multi-user MIMO precoding matrices," IEEE Trans. Wireless Commun., vol. 7, no. 3, pp. 953-961, Mar. 2008

[9] B. Hochwald, C. Peel and A. Swindlehurst, "A vector-perturbation technique for near capacity multiantenna multiuser communication - Part II: Perturbation," IEEE Trans. Commun., vol. 53, no. 3, Mar. 2005.

[10] M. Costa, "Writing on dirty paper," IEEE Trans. Inform. Theory, vol. 29, no. 3, pp. 439-441, May 1983.

[11] A. Khina and U. Erez, "On the Robustness of Dirty Paper Coding," IEEE Trans. Commun., vol. 58, no. 5, May 2010.

[12] M. Tomlinson, "New automatic equaliser employing modulo arithmetic," Electronic Letters, vol. 7, Mar. 1971.

[13] H. Harashima and H. Miyakawa, "Matched-transmission technique for channels with intersymbol interference," IEEE Trans. Commun., vol. 20, Aug. 1972.

[14] R. Fischer, C. Windpassinger, A. Lampe and J. Huber, "Space-time transmission using Tomlinson-Harashima precoding," in Proc. ITG Conf. on Source and Channel Coding (SCC), Berlin, Jan. 2002, pp. 139-147.

[15] W. Y, D. Varodayan and J. Cioffi, "Trellis and convolutional precoding for transmitter based interference presubstration," IEEE Trans. Commun., pp. 1220-1230, Jul. 2005.

[16] U. Erez, S. Shamai and R. Zamir, "Capacity and lattice strategies for cancelling known interference," IEEE Trans. Inf. Theory, pp. 3820-3833, Nov. 2005.

[17] C. Windpassinger, R. Fischer, T. Vencel and J. Huber, "Precoding in multiantenna and multiuser communications," IEEE Trans. Wireless Commun., vol. 3, no. 4, Jul. 2004.

[18] C. Windpassinger, T. Vencel, and R. Fischer, "Precoding and loading for BLAST-like systems," in Proc. IEEE Int. Conf. on Commun. (ICC), Anchorage, Alaska, USA, May 2003.

[19] M. Joham, J. Brehmer, and W. Utschick, "MMSE approaches to multiuser spatio-temporal Tomlinson-Harashima precoding," in Proc. 5th ITG Conf. Source and Channel Coding (SCC), Germany, Jan. 2004.

[20] M. Joham and W. Utschick, "Ordered spatial Tomlinson-Harashima precoding," in Smart Antennas-State-of-the-Art, ser. EURASIP Book Series on Signal Processing and Communications. New York: Hindawi Publishing Corporation, 2005.

[21] D. Wübben, J. Rinas, R. Böhnke, V. Kühn and K. Kammeyer "Efficient algorithm for detecting layered space-time codes," in Proc. ITG Conf. on Source and Channel Coding (SCC), Berlin, Germasy, Jan. 2002, pp. 399-405.

[22] D. Wübben, R. Böhnke, V. Kühn and K. Kammeyer, "MMSE extension of V-BLAST based on sorted QR decomposition," in Proc. IEEE Vehicular Technology Conf. (VTC) Fall, Orlando, Florida, USA, Oct. 2003.

[23] J. Liu and W. Krzymień, "Improved Tomlinson-Harashima precoding for the downlink of multi-user MIMO systems," Canadian Journal of Electrical and Computer Engineering, vol. 32, Summer 2007.
[24] R. Habendorf and G. Fettweis, "On ordering optimization for MIMO systems with decentralized receivers," in Proc. IEEE Vehicular Technology Conf. (VTC) Spring, Melbourne, Australia, May 2006, pp. 18441848.

[25] J. Liu and W. Krzymień, "A Novel nonlinear joint transmitter-receiver processing algorithm for the downlink of multiuser MIMO systems," IEEE Trans. Veh. Technol., vol. 57, no. 4, Jul. 2008

[26] C. Fung, W. Yu and T. Lim, "Precoding for the multiantenna downlink: multiuser SNR gap and optimal user ordering," IEEE Trans. Commun., vol. 55, no. 1, Jan. 2007.

[27] N. Dào and Y. Sun, "User-selection algorithms for multiuser precoding," IEEE Trans. Veh. Technol., vol. 59, no. 7, Sep. 2010.

[28] V. Stankovic and M. Haardt, "Successive Optimization TomilinsonHarashima Precoding (SO-THP) for Multi-user MIMO systems," in Proc. IEEE Int. Conf. on Acoustics, Speech, and Signal Processing (ICASSP), Philadelphia, PA, Mar. 2005, pp. 1117-1120.

[29] K. Zu, R. C. de Lamare and M. Haardt, "Multi-Branch TomlinsonHarashima precoding for single-user MIMO systems," in Proc. ITG/IEEE Workshop on Smart Antennas, Dresden, Germany, Mar. 2012.

[30] M. Huang, S. Zhou and J. Wang, "Analysis of Tomlinson-Harashima precoding in multiuser MIMO systems with imperfect channel state information," IEEE Trans. Veh. Technol., vol. 57, no. 5, Sep. 2008.

[31] D. Shiu and J. Kahn, "Layered Space-Time Codes for Wireless Communications using Multiple Transmit Antennas," in Proc. IEEE Int. Conf. on Communications (ICC'99), Vancouver, B.C., Canada, Jun. 1999.

[32] U. Abay and R. Fischer, "Comparison of generalized TomlinsonHarashima precoding strategies for the broadcast channel," in Proc. ITG/IEEE Workshop on Smart Antennas, Aachen, Germany, Feb. 2011.

[33] F. Dietrich, P. Breun and W. Utschick, "Robust Tomlinson-Harashima precoding for the wireless broadcast channel," in IEEE Trans. Signal Process., vol. 55, no. 2, Feb. 2007

[34] R. C. de Lamare and R. Sampaio-Neto, "Minimum mean squared error iterative successive parallel arbitrated decision feedback detectors for DS-CDMA systems," IEEE Trans. Commun., May 2008.

[35] R. Fa and R. C. de Lamare, "Multi-branch successive interference cancellation for MIMO spatial multiplexing systems: design, analysis and adaptive implementation," IET Commun., May 2010.

[36] Y. Cai, R. C. de Lamare, R. Fa, "Switched interleaving techniques with limited feedback for interference mitigation in DS-CDMA systems," IEEE Trans. Commun., vol. 59, no. 7, Jul. 2011

[37] R. C. de Lamare, "Adaptive and Iterative Multi-Branch MMSE Decision Feedback Detection Algorithms for Multi-Antenna Systems," IEEE Trans. Wireless Commun., vol.12, no.10, pp.5294-5308, October 2013.

[38] G. Golub and C. Van Loan, Matrix Computations. The Johns Hopkins University Press, 1996.

[39] K. Zu and R. C. de Lamare, "Low-complexity lattice reduction-aided regularized block diagonalization for MU-MIMO systems," IEEE Commun. Lett., 2012.

[40] K. Zu, R. C. de Lamare and M. Haardt, "Generalized design of low-complexity block diagonalization type precoding algorithms for multiuser MIMO systems," IEEE Trans. Commun., vol. 61, no. 10, Oct. 2013.

[41] K. Kusume, M. Joham, W. Utschick and G. Bauch, "Cholesky factorization with symmetric permutation applied to detecting and precoding spatially multiplexed data streams," IEEE Trans. Signal Process., vol. 55 , no. 6, Jun. 2007.

[42] D. Schmidt, M. Joham and W. Utschick, "Minimum mean square error vector precoding," European Trans. on Telecommun., vol. 19, no. 3, 2008.

[43] B. Hassibi and H. Vikalo, "On the sphere decoding algorithm: Part I the expected complexity," IEEE Trans. Signal Process., vol. 53, no. 8, pp. 2806-2818, Aug. 2005.

[44] K. Zu and R. C. de Lamare, "Pre-sorted multiple-branch successive interference cancelation detection for high-dimensional MIMO systems," in Proc. ITG/IEEE Workshop on Smart Antennas, Dresden, Germany, Mar. 2012.

[45] A. Paulraj, R. Nabar and D. Gore, Introduction to space-time wireless communications. Cambridge University Press, 2003.

[46] K. Zu, R. C. de Lamare and M. Haardt, "Lattice reduction-aided regularized block diagonalization for multiuser MIMO systems", IEEE Wireless Commun. and Networking Conf. (WCNC), Paris, France, Apr. 2012.

[47] S. Loyka, "Channel capacity of MIMO architecture using the exponential correlation matrix," IEEE Commun. Lett., vol. 5, no. 9, pp. 369-371, Sep. 2001. 